\title{
Simulation in Neuroanesthesia: Need of the Hour in India
}

\author{
Rajiv Chawla ${ }^{1} \quad$ Akhilesh Pahade ${ }^{1}$ \\ ${ }^{1}$ Department of Anaesthesiology and Critical Care, Rajiv Gandhi \\ Cancer Institute and Research Centre, Delhi, India
}

J Neuroanaesthesiol Crit Care 2018;5:184-186

\begin{abstract}
Address for correspondence Akhilesh Pahade, MD (Anaesthesiology), PDCC (Cardiac Anaesthesiology), Department of Anaesthesiology and Critical Care, Rajiv Gandhi Cancer Institute and Research Centre, Rohini, Sector 5, Delhi 110085, India (e-mail: akhildada09@yahoo.in).
\end{abstract}

"I hear and I forget. I see and I remember. I do and I understand." Confucius. ${ }^{1}$

India has the unenviable distinction of being the capital of Traumatic Brain Injury (TBI) and stroke. In India, more than 100,000 lives are lost every year with over 1 million suffering from serious head injuries. One out of six trauma victims in India die while in the United States, this figure is 1 out of $200 .^{2}$ This clearly highlights the lacunae in our existing healthcare system. The burden of stroke in India is also increasing at an alarming rate. Over the past decade, the cumulative stroke incidence ranging from 105 to $152 / 100,000$ persons. ${ }^{3}$ In both the conditions (stroke and TBI), early intervention improves the outcome immensely. Ironically, for a population of 1.35 billion, there are only 1,360 qualified neurosurgeons and approximately 700 neuroanesthesiologists. ${ }^{4-6}$ They are mainly restricted to major cities. The population in the rural areas, which accounts for $70 \%$ of Indian population is left unattended. In the interest of humanity, the need of the hour is to have trained personnel who can intervene early and provide timely care-and we need to have them fast!! Training by simulation seems to be the answer for our current woes and high demand of trained motivated manpower.

Simulation as applied to healthcare has been defined as "a technique, not a technology, to replace or amplify real-life experiences that evoke or replicate substantial aspects of real world in a fully interactive manner." In 2014, a national scoping report published by the Association for Simulated Practice in Healthcare (ASPiH) explored the spectrum of uses of simulation in healthcare training in the UK and concluded that it is clear that simulation-based education is now established as an important tool in the armamentarium for training and assessing healthcare practitioners. ${ }^{7}$ Review of literature reveals that simulation has an important role in upgrading skills and knowledge in various fields of neuromedicine. Scope of simulation in neurosurgery, neuroanesthesia, and critical care is immense with the effects far reaching and beneficial for the general population who does not have proper access to medical and surgical facilities. Not only do these simulation programs shorten the learning curve but also help to reduce the response time in identifying and tackling critical and error-prone situations effectively,

received

July 30, 2018 accepted after revision

September 21, 2018

published online

October 16, 2018 thereby providing great help in rapid decision making in uncertain situations. They are also effective in reinforcing the learnt skills and help in taking trauma and stroke ready hospitals close to those who need them the most.

Managing crisis situation requires a common curriculum along with a uniform access to simulation techniques and programs. The available simulation training programs for TBI and stroke are seen to primarily consist of two components. The first one is teaching non-technical skills. Nontechnical skills are cognitive, social, and personal resource skills that complement technical skills. Non-technical skills are achieved by role plays and contribute to safe and efficient task performance. The second component comprises simulators, such as mannequins and virtual reality equipment, which upgrade the skills.

With advancements in technology, there has been major developments and upgradation in simulators. The recent ones can be programmed to mimic multiple real-life clinical situations. Simulation training programs are structured programs, a combination of lectures and skill development hands-on training. Specific case scenarios such as cardiac arrest in the prone or lateral decubitus, venous air embolism, subarachnoid hemorrhage-associated vasospasm, raised intracranial pressure in the intensive care patient stroke, cerebral artery stenosis, head trauma, hypercapnia, hypotension, cerebral autoregulation, and thrombolytic therapy and different types of head injuries can be played out. Intracranial pressures and transcranial Doppler's monitoring can be demonstrated. Futuristic simulation courses include case overviews, patient history, neurologic exam results, simulation parameters and expected actions, mock CT (computerized tomography) scans and laboratory results, and supporting literature references. ${ }^{8,9}$ Simulation of such events allow operating room (OR) staff to identify and practice a predetermined role and minimize human error. It paves way for doctors, nurses, and technician alike, to get trained to work in the neurosurgical unit. ${ }^{8}$

Laerdal SimMan 3G simulator helps to demonstrate eye signs, convulsions along with features such as cardiopulmonary resuscitation, vascular access, bleeding and drug
Copyright $\odot 2018$ Indian Society of Neuroanaesthesiology and Critical Care
License terms

$10.1055 / \mathrm{s}-0038-1675326$ ISSN 2348-0548.

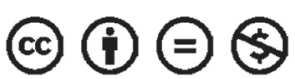


recognition..$^{10}$ NewroSim simulator allows online visualization of cerebrovascular/hemodynamic variables involved in cerebral autoregulation and allows the user to assess the impact of therapeutic intervention. ${ }^{9}$ Pharmacological/ mechanical recanalization simulator may prove to be the game changer in acute ischemic stroke.

One brain simulation program combines lectures on neurosurgery, neuroanesthesia, and anesthesia for interventional neuroradiology and exposes those enrolled to mock drills on head injuries and other neurological emergencies, which include management of patients with head injury, venous air embolism, subarachnoid hemorrhage-associated vasospasm, raised intracranial pressure in the intensive care patient, and transfer to definitive care center. ${ }^{11}$

Neurotrauma Moulage is a traumatic brain injury simulator. It incorporates a very approximate mathematic model of neurotrauma physiology. It is designed to simulate a range of conditions affecting the management of the injured brain, and to encourage a greater understanding of the main tenets of traumatic brain injury management-especially the prevention of secondary injury. This simulator can also be accessed online and is available in public domain. ${ }^{12}$

Multimedia three-dimensional (3D) printing has enabled fabricating high-fidelity, multitexture models for planning and training of operative procedures. ${ }^{13}$

Various neurosimulation programs/courses are now being conducted in developed countries. In India, earliest efforts in neurosurgical simulation were taken by AIIMS (All India Institute of Medical Sciences), New Delhi by establishing Neurosurgery Education And Training School (NETS). ${ }^{14}$ Neuroanaesthesia update AIIMS 2016 also highlighted the importance of simulation in neuroanesthesia by demonstrating management of refractory intracranial pressure in ICU (intensive care unit) through a skit. ${ }^{15}$ Another premier government institute, NIMHANS is also conducting regular training programs and courses to bridge the current gap. NIMHANS has also conducted studies on TBI and stroke. ${ }^{16}$ It also has a virtual learning center to deal with addictions in the community. ${ }^{17}$ We need similar initiatives in the field of stroke and TBI. Corporate hospitals such as Medanta, The Medicity, have also stepped in to contribute in the field of simulation and training and are regularly conducting neuroanesthesia training and simulation programs. ${ }^{18}$

The Indian Society of Neuroanaesthesiology and Critical Care (ISNACC) conducts regular conferences and workshops, and supports fellowship programs to not only strengthen the skills of existing neuroanesthesiologists but also adds to the number of trained personnel. ISNACC has taken the onus of introducing simulation-based workshops in the conferences being conducted. ${ }^{19}$

One of the major limitations is the need for a common and standardized curriculum, and, without a standard assessment criterion, implementing and developing a training curriculum will be incomplete. Simulators may not replicate operating room conditions effectively.

Even though the field of healthcare in India is developing by leaps and bounds, cost constraints exist, with the cost of virtual and mechanical simulators keeping them out of reach of many research institutes. In addition, even though a start has been made in the form of NETS (by AIIMS), similar efforts are needed across institutes all over the country at affordable cost to ensure adequate penetration. This would require financial support from government and private sector (in form of corporate and social responsibility [CSR]). There should also be some incentive for those getting trained. Professional societies such as ISNACC should consider this as their social responsibility and should take lead by conducting frequent, uniformly, and scientifically structured simulation programs on similar lines as the successful, well publicized, and popular cardiopulmonary resuscitation (CPR) certification programs. Emphasis should also be laid on Advanced Trauma Life Support (ATLS) training for all medical and paramedical staff.

The greatest challenge that we face today regarding simulation-based education and training is its widespread adoption and sustainability in an increasingly fiscally constrained healthcare system. It is also often seen that the enthusiasm for adopting a new training modality is high, but with time this initial momentum loses steam and one tends to be drifted to yet another newer modality appearing on the scene. Progressive attention to patient salvage and survival has helped anesthesia and especially neuroanesthesia to embrace the concept and science of simulation. Fields of engineering and computer technology are providing us with advanced, cost-effective solutions to support simulation as a tool for training. Taking simulation to the workplace should be the norm and needs to be harnessed as a force to improve the design and delivery of high-quality, safe patient care. ${ }^{7} \mathrm{We}$ are perfectly placed to harness the benefits of advances in simulation through collaborative research into training interventions and systems design that demonstrate a clear impact on patient outcomes and organizational resilience. ${ }^{7}$ It is vital that we continue our journey in this domain as futuristic simulation programs. High-fidelity simulators are bound to play an important cog in the wheel in the current scenario and in days ahead, but the key to success lies in sustained, time bound, and effective implementation while providing greater availability of training facilities and eventually trained personnel in peripheries.

\section{Conflict of Interest}

None.

\section{References}

1 Vaillancourt R. "I hear and I forget, I see and I remember, I do and I understand". Can J Hosp Pharm 2009;62(4):272-275

2 Traumatic brain injury. Available at: http://indianheadinjuryfoundation.org/traumatic-brain-injury/. Accessed July 22, 2018

3 Kamalakannan S, Gudlavalleti ASV, Gudlavalleti VSM, Goenka S, Kuper H. Incidence \& prevalence of stroke in India: a systematic review. Indian J Med Res 2017;146(2):175-185

4 Misra BK, Singh VP. Neurosurgery in India. AANS Neurosurgeon 2017;26(1). Available at: http://aansneurosurgeon.org/inside -neurosurgeon/neurosurgery-in-india/

5 Indian population: retrieving data. Available at: https:// www.worldometers.info/world-population/india-population/ Accessed July 22, 2018 
6 Member directory Indian Society of Neuroanaesethesia and critical care. Available at: http://www.isnacc.org/membership_directory.html. Accessed on July 22, 2018

7 Higham H, Baxendale B. To err is human: use of simulation to enhance training and patient safety in anaesthesia. $\mathrm{Br} \mathrm{J}$ Anaesth 2017;119(Suppl 1) :i106-i114

8 Bhatt R, Khanna P. Simulation in neuroanesthesia: how much to learn? J Neuroanaesth Crit Care 2018;05(2):83-86

9 NewroSim $^{\mathrm{TM}}$ traumatic brain injury and stroke care training solution. Available at: https://www.gaumard.com/newrosim. Accessed July 22, 2018

10 Helping improve patient outcome: SimMan 3G. Available at: https://www.laerdal.com/in/products/simulation-training/ emergency-care-trauma/simman-3g/Accessed July 22, 2018

11 One brain neuroanaesthesia. Available at: http://www.onebrain.org.uk/. Accessed July 22, 2018

12 Neurotrauma moulage. Available at: http://www.trauma.org/ resus/neuromoulage/index.html: Accessed July 22, 2018

13 Hoang D, Perrault D, Stevanovic M, Ghiassi A. Surgical applications of three-dimensional printing: a review of the current literature \& how to get started. Ann Transl Med 2016;4(23):456
14 Suri A, Patra DP, Meena RK. Simulation in neurosurgery: past, present, and future. Neurol India 2016;64(3):387-395

15 Singh GP, Dube SK, Chaturvedi A. Report of AIIMS neuroanaesthesia update 2016. J Neuroanaesth Crit Care 2017;4:61-63

16 Traumatic brain injury-NI MHNS. Available at: http://www. nimhans.ac.in/sites/default/files/Traumatic\%20Brain\%20Injury_0.pdf. Accessed July 22, 2018

17 Virtual knowledge network NIMHANS: centre for addiction medicine, Dept. of Psychiatry. Available at: vlc.nimhans.ac.in.: Accessed July 22, 2018

18 Annual Medanta neurocritical care conference workshops. Available at: http://www.mncc.co.in/workshop.html. Accessed July 22, 2018

19 20th annual conference of the Indian Society of Neuroanaesthesiology and Critical care. Available at: http://www. isnacc2019.com/registration.html. Accessed July 22, 2018 